\title{
Thromboelastography-Guided Management of Anticoagulated COVID-19 Patients to Prevent Hemorrhage
}

\author{
John E. Stillson, BS ${ }^{1}$ Connor M. Bunch, BS ${ }^{1}$ Laura Gillespie, PharmD ${ }^{2}$ Rashid Khan, MD $^{3}$ \\ Meredith Wierman, DO ${ }^{4}$ Joseph Pulvirenti, MD ${ }^{4}$ Htay Phyu, MD ${ }^{4}$ Stephen Anderson, MD ${ }^{5,6}$ \\ Mahmoud Al-Fadhl, BS ${ }^{6}$ Anthony V. Thomas, BS ${ }^{6}$ Hau C. Kwaan, MD ${ }^{7}$ Ernest Moore, MD ${ }^{8}$ \\ Hunter Moore, MD ${ }^{8}$ Mark M. Walsh, MD ${ }^{5,6}$
}

1 South Bend Campus, Indiana University School of Medicine, South Bend, Indiana

2 Department of Quality and Performance Improvement, Saint Joseph Regional Medical Center, Mishawaka, Indiana

${ }^{3}$ Department of Hematology, Michiana Hematology Oncology, Mishawaka, Indiana

${ }^{4}$ Department of Infectious Disease, Saint Joseph Regional Medical Center, Mishawaka, Indiana

${ }^{5}$ Department of Emergency Medicine, Saint Joseph Regional Medical Center, Mishawaka, Indiana

${ }^{6}$ Department of Internal Medicine, Saint Joseph Regional Medical Center, Mishawaka, Indiana

${ }^{7}$ Division of Hematology and Oncology, Northwestern University Feinberg School of Medicine, Chicago, Illinois

${ }^{8}$ Department of Surgery, Denver Health Medical Center and University of Colorado Health Sciences Center, Denver, Colorado

Semin Thromb Hemost 2021;47:442-446.

Major hemorrhage is an underreported complication of hospitalized and anticoagulated patients infected by severe acute respiratory syndrome coronavirus 2 (SARS-CoV-2) (i.e., the pathogen causing novel coronavirus disease 2019 [COVID-19]). Recent evidence demonstrates some degrees of hypercoagulability in these patients, prompting an expanding group of institutions to establish guidelines recommending intermediate or therapeutic doses of low-molecular weight heparin (LMWH) or unfractionated heparin (UFH) for COVID-19 patients with moderate to severe pneumonia. ${ }^{1-7}$ Monitoring hemostatic competence of COVID-19 patients is quite challenging, because their initial hypercoagulability can be quickly supplanted by hypocoagulability in the presence of anticoagulation or even hemostasis exhaustion. $^{1,8}$

The prediction of bleeding for critically ill COVID-19 patients on intermediate or therapeutic anticoagulation remains an area of new investigation. ${ }^{9}$ Therefore, it was considered beneficial to determine the clinical use of point-of-care thromboelastography (TEG) when accompa-

published online February 19, 2021
Issue Theme Maintaining Hemostasis and Preventing Thrombosis in COVID-19 -Part II; Guest Editors: Emmanuel J. Favaloro, PhD, FFSc (RCPA) and Giuseppe Lippi, MD.

Address for correspondence Mark M. Walsh, MD, Department of Internal Medicine, Saint Joseph Regional Medical Center, 5215 Holy Cross Parkway, Mishawaka, IN 46545

(e-mail: markwalshmd@gmail.com).

nied by common coagulation tests (CCTs)-prothrombin time (PT), activated partial thromboplastin time (aPTT), fibrinogen, D-dimer, and platelet count-in predicting risk of bleeding. $^{4-8}$ The CCTs have not been shown to reliably predict bleeding events in COVID-19 patients. TEG and rotational thromboelastometry (ROTEM) have recently been used in small studies to guide anticoagulation therapy in these patients. ${ }^{3,10-12}$ Therefore, we elected to use TEG as an accessory to CCTs to predict bleeding as defined by a World Health Organization (WHO) bleeding scale score $\geq 2$ for COVID-19 intensive care unit (ICU) patients who received intermediate or therapeutic anticoagulation. ${ }^{13}$

This protocol development was approved by the medical executive/quality assurance committees of Saint Joseph Regional Medical Center at Mishawaka, IN. All patients were aged $\geq 18$ years with confirmed COVID-19 (defined as a positive SARS-CoV-2 reverse transcriptase polymerase chain reaction test by nasopharyngeal/oropharyngeal swab or sputum specimen or by positive SARS-CoV-2 IgM antibody). Data were obtained prospectively by the COVID-19

(c) 2021. Thieme. All rights reserved. Thieme Medical Publishers, Inc., 333 Seventh Avenue, 18th Floor, New York, NY 10001, USA
DOI https://doi.org/ 10.1055/s-0041-1723754. ISSN 0094-6176. 
coagulation committee in daily review of all admissions to the hospital who received infectious disease consultation. The dates of data collection were April 26, 2020 to September 15, 2020. Patient data collected includes age, gender, comorbidities, clinical description of bleeding events, laboratory tests, and anticoagulation medication administered. PT and aPTT were performed with the Sysmex CA-1500 and reagents Innovin and $\mathrm{CaCl}_{2}$. Fibrinogen was quantified in samples mixed with thrombin and Owren's Veronal buffer. D-dimer was quantified as fibrinogen equivalent units with the Innovance D-dimer Assay. Platelets were counted with a Sysmex XP-2000 (all products from Siemens Medical Solutions, Malvern, PA). TEG data were obtained with the TEG5000 hemostasis analyzer (Haemonetics, Braintree, MA). This protocol development includes TEG data of samples mixed only with citrated kaolin and without heparinase. TEG parameters reaction time $(R)$, clot formation time $(k), \alpha$ angle, maximum amplitude, and lysis at 30 minutes (LY30), and CCTs were measured throughout the patients' hospitalizations. TEG parameter LY30 maintained a reading of less than $1 \%$ throughout our protocol development for nearly all patients and was thus not included in our analysis.

Of 222 consecutive admissions from April 26, to September 15,2020 , only anticoagulated COVID-19 patients who were admitted to the ICU and met the criteria of two predetermined groups, bleeding or nonbleeding, were included in the analysis. Patients were excluded for BMI $>50$ $\mathrm{kg} / \mathrm{m}^{2}$. The bleeding group was defined by patients who bled while receiving intermediate or therapeutic UFH. Bleeding was defined by a WHO bleeding scale score $\geq 2 .{ }^{13}$ There were 10 patients who met the criteria of the bleeding group. Two of these 10 patients had both gastrointestinal bleed and hemothorax during hospitalization $(n=2)$. The remaining eight of 10 bleeding patients were characterized by a singular bleed of hemothorax $(n=1)$, gastrointestinal $(n=1)$, intramuscular $(n=3)$, retroperitoneal $(n=1)$, or at vascular access site for dialysis $(n=2)$.

The nonbleeding group comprised 21 COVID-19 ICU patients who received intermediate or therapeutic doses of intravenous UFH or subcutaneous enoxaparin and did not have a bleeding event as defined by a WHO bleeding score $\geq 2$. To reduce exposure to COVID-19 by medical personnel, enoxaparin was first chosen for many patients in the nonbleeding group due to its easier administration compared with UFH, which demands frequent laboratory assessment and dose adjustments. 5,14

There was no statistically significant difference in the demographics or blood products received between the two groups. There was a statistically significant difference in the rates of thromboses, surgical intervention, invasive ventilation, and expiration between these two groups. Surgical interventions in the bleeding group included embolectomy, thoracostomy, and bladder irrigation (-Table $\mathbf{1}$ ).

Prior to September 16, 2020, initial therapeutic anticoagulation for UFH was defined as 60 units/ $\mathrm{kg}$ bolus, followed by 12 units $/ \mathrm{kg} / \mathrm{h}$ infusion. Initial intermediate anticoagulation for UFH was defined as infusion of 12 units $/ \mathrm{kg} / \mathrm{h}$ without bolus infusion. For enoxaparin, initial therapeutic anticoa- gulation was defined as $1 \mathrm{mg} / \mathrm{kg}$ subcutaneous every 12 hours. Initial intermediate anticoagulation for enoxaparin was defined as $0.6 \mathrm{mg} / \mathrm{kg}$ subcutaneous every 12 hours. Initial anticoagulation for UFH was guided by standard published algorithms for aPTT. After this beginning period of patient analysis, an increased number of patients with and without documented thrombosis were noted to have clinically significant bleeds which prompted modification of our UFH dosing guidelines. Beginning on September 16, until December 1, 2020, all patients on intravenous UFH were managed according to a modified bolus-free reduced dosage (-Fig. 1). Thereafter, UFH, platelets, and fibrinogen were administered based on recent clinical recommendations for COVID-19 patients in the ICU, clinical evaluation by the authors, and on a TEG/aPTT-guided algorithm (-Table 2). ${ }^{4,6,7,15-18}$

In the nonbleeding group, the averages of each parameter throughout hospitalization were calculated to provide a reference range for analysis, which was then compared with the value on the day of bleeding in the bleeding group (-Table 3). The laboratory data shown in -Table 3 was collected from April 26 to September 15, 2020, prior to the final establishment of our formal TEG/aPTT-guided anticoagulation algorithm. The TEG parameters and the CCTs were analyzed for statistical correlation with bleeding using the Mann-Whitney test. Results are reported in medians, interquartile ranges, and $p$-values. A value of $p<0.05$ was the threshold for statistical significance.

The parameters which predicted bleeding compared with the nonbleeding group were the $R(p=0.0001), k$ $(p=0.0002), \alpha$ angle $(p=0.0001)$, PT $(p=0.0013)$, aPTT $(p=0.0006)$, and fibrinogen $(p=0.0019)$ (-Table 3$)$. For the bleeding group, the average number of days from admission to day of bleeding was $8.8 \pm 3.8$ days. The number of patients on intermediate and therapeutic UFH doses before (April 26 to September 15, 2020) and after (September 16 to December 1, 2020) the establishment of a reduced nonbolus protocol for intermediate and therapeutic UFH revealed that 10 of 35 (0.286, Agresti-Coull 95\% CI: 0.162-0.452) patients bled prior to the establishment of the nonbolus protocol, versus 1 of 51 ( 0.020 , Agresti-Coull 95\% CI: -0.006 to 0.113 ) afterward. With the nonbolus protocol, patients had 20 -fold decreased likelihood of hemorrhage (odds ratio; 95\% CI: 2.4-165.1, $p<0.001$ by Fisher's exact test) compared with patients treated prior to the establishment of this protocol.

Hemorrhage in hospitalized and anticoagulated COVID19 patients has been reported in small studies. A United Kingdom series of 66 COVID-19 ICU patients on standard thromboprophylaxis showed an $11 \%$ incidence of major hemorrhage. ${ }^{19}$ Another French study of 92 patients on prophylactic or therapeutic anticoagulation demonstrated a $21 \%$ incidence of major hemorrhage. ${ }^{20}$ The largest study to date on anticoagulated COVID-19 patients and bleeding risk was a single-center United States retrospective study that demonstrated an $11 \%$ bleeding rate for 102 patients treated with therapeutic dosing, which also showed a statistically significant correlation to inpatient death. ${ }^{21}$ Overall, the literature on major hemorrhage in hospitalized, 
Table 1 Demographics table of the bleeding and nonbleeding groups

\begin{tabular}{|l|l|l|l|}
\hline & Bleeding group $(\boldsymbol{n}=\mathbf{1 0})$ & Nonbleeding group $(\boldsymbol{n}=\mathbf{2 1})$ & $\boldsymbol{p}$-Value \\
\hline Age (years), mean (SD) & $59.4(17.5)$ & $65.2(17.5)$ & 0.404 \\
\hline BMI (kg/m $\left.{ }^{2}\right)$, median (IQR) & $33.5(7.8)$ & $31.2(10.9)$ & 0.263 \\
\hline Female, $n$ (\%) & $4(40.0 \%)$ & $7(33.3 \%)$ & 0.717 \\
\hline Caucasian, $n$ (\%) & $7(70.0 \%)$ & $8(38.1 \%)$ & 0.097 \\
\hline Comorbidities, $n$ (\%) & & & \\
\hline Morbid obesity & $4(40.0 \%)$ & $7(33.3 \%)$ & 0.717 \\
\hline Hypertension & $8(80.0 \%)$ & $14(66.7 \%)$ & 0.445 \\
\hline Hyperlipidemia & $7(70.0 \%)$ & $7(33.3 \%)$ & 0.055 \\
\hline Coronary artery disease & $1(10.0 \%)$ & $4(19.0 \%)$ & 0.261 \\
\hline Heart failure & $3(30.0 \%)$ & $2(9.5 \%)$ & 0.073 \\
\hline Type II diabetes mellitus & $4(40.0 \%)$ & $8(38.1 \%)$ & 0.919 \\
\hline Renal failure & $3(30.0 \%)$ & $1(4.8 \%)$ & 0.050 \\
\hline Immunosuppression & $2(20.0 \%)$ & $4(19.0 \%)$ & 0.950 \\
\hline Chronic obstructive pulmonary disorder & $3(30.0 \%)$ & $6(28.6 \%)$ & 0.935 \\
\hline Tobacco use & $2(20.0 \%)$ & $3(14.3 \%)$ & 0.686 \\
\hline DVT during hospitalization & $2(20.0 \%)$ & $0(0.0 \%)$ & 0.034 \\
\hline Blood products, $n$ (\%) & & & \\
\hline Packed red cells & $5(50.0 \%)$ & $5(23.8 \%)$ & 0.145 \\
\hline Cryoprecipitate & $6(60.0 \%)$ & $6(28.6 \%)$ & 0.093 \\
\hline Platelets & $3(30.0 \%)$ & $2(9.5 \%)$ & 0.147 \\
\hline Fresh frozen plasma & $8(80.0 \%)$ & $13(61.9 \%)$ & 0.314 \\
\hline Interventions, $n$ (\%) & & $1(4.8 \%)$ & 0.013 \\
\hline Surgical & $4(40.0 \%)$ & $1(4.8 \%)$ & 0.003 \\
\hline Invasive ventilation & $5(50.0 \%)$ & $0(0.0 \%)$ & 0.034 \\
\hline Expired, $n$ (\%) & $2(20.0 \%)$ & & \\
\hline
\end{tabular}

Abbreviations: BMI, body mass index; DVT, deep vein thrombosis; IQR, interquartile range; $n$, sample size; SD, standard deviation.

Note: There is a statistically significant difference in thromboses, interventions, invasive ventilation, and expiration rate between the two groups.

anticoagulated COVID-19 patients is sparse. Yet, this is a relatively common and lethal complication that requires better monitoring of anticoagulation to prevent hemorrhage.

Critically ill COVID-19 patients in the ICU who do not have established macrovascular thromboses may be offered varying intensities of anticoagulation, ranging from standard prophylaxis to full therapeutic anticoagulation given the heterogeneity of recommendations since the beginning of this pandemic. There is little guidance in the literature regarding algorithms to assist in dosing UFH or LMWH for this group of patients. We suggest that, in this environment of disparate recommendations regarding the intensity of anticoagulation in this subset of patients, the use of the TEG and aPTT allows for directed UFH titration, which may prevent bleeding complications. Without evidence-based medicine to guide physicians during this unique pandemic, our experience may justify the empirical choice of an intermediate dose of UFH. It has been proposed that an intermediate dose of UFH is worthwhile because it possesses an ability to act as an anti-inflammatory agent at the level of the endothelium as well as the ability to interact with and bind to the spike protein on COVID-19 viral particles. ${ }^{1,2,4,6,8}$

The findings of this protocol development reveal the complexity of the initially hypercoagulable state of critically ill COVID-19 patients. We have found that TEG is useful in guiding goal directed UFH therapy due to its narrow therapeutic window when given to COVID-19 patients. We propose that this narrow therapeutic window resembles the spectrum of coagulopathies occurring in patients on extracorporeal membrane oxygenation who require careful personalized titration, not just with aPTT, but also with TEG and anti-Xa levels. $^{22-24}$

A limitation of this protocol development is the small sample of 31 COVID-19 ICU patients. Yet, when compared with many of the papers that have been published concerning the utilization of the TEG and ROTEM in the COVID-19 population, this is a relatively high number of patients. ${ }^{3,10-12}$ Another limitation of this study was that anti-Xa levels were not used to monitor therapeutic levels of LMWH or UFH, because anti-Xa levels were not available around the clock at 


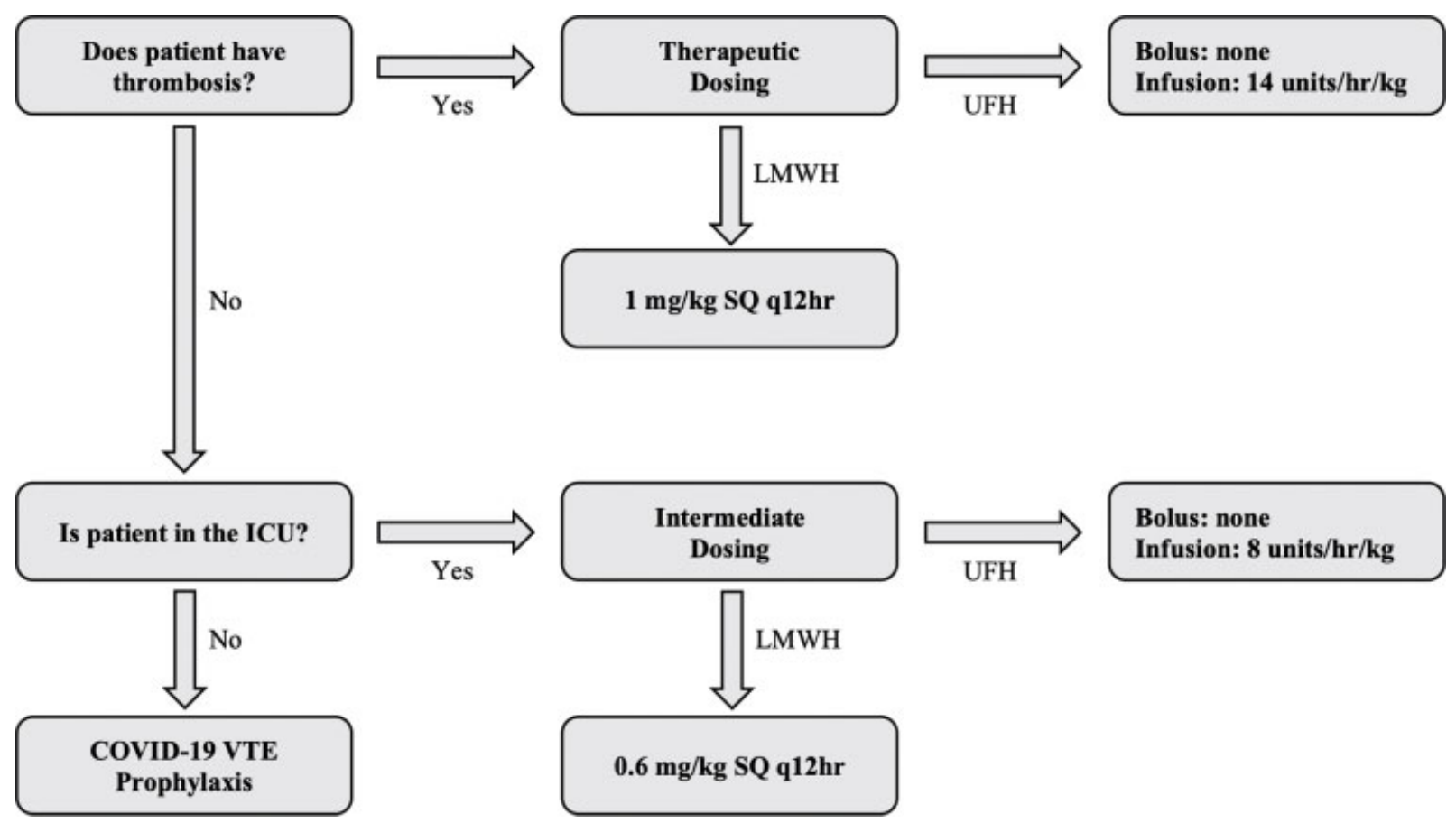

Fig. 1 Nonbolus anticoagulation dosing protocol for COVID-19 patients after September 16, 2020. COVID-19, coronavirus disease 2019; ICU, intensive care unit; LMWH, low-molecular weight heparin; q12hr, every twelve hours; SQ, subcutaneous; UFH, unfractionated heparin; VTE, venous thromboembolism.

Table 2 TEG/aPTT sample algorithm for anticoagulated COVID19 ICU patients

\begin{tabular}{|l|l|}
\hline TEG/CCT reading & Action \\
\hline $\mathrm{R}$ kaolin $=20-25 \mathrm{~min}$ & Reduce heparin dose by $10 \%$. \\
\hline $\mathrm{R}$ kaolin $=13-20 \mathrm{~min}$ & None \\
\hline $\mathrm{R}$ kaolin $<13 \mathrm{~min}$ & Increase heparin dose by $10 \%$. \\
\hline $\mathrm{MA}<50 \mathrm{~mm}$ & Reduce heparin dose by $10 \%$. \\
\hline $\mathrm{MA}<40 \mathrm{~mm}$ & Hold heparin, reevaluate patient. \\
\hline $\begin{array}{l}\text { Fibrinogen } \\
<200 \mathrm{mg} / \mathrm{dL}\end{array}$ & $\begin{array}{l}\text { Give } 5 \text { units cryoprecipitate } \\
\text { (raise fibrinogen } 25-50 \mathrm{mg} / \mathrm{dL} \text { ). }\end{array}$ \\
\hline $\begin{array}{l}\text { Fibrinogen } \\
<150 \mathrm{mg} / \mathrm{dL}\end{array}$ & $\begin{array}{l}\text { Give } 10 \text { units cryoprecipitate } \\
\text { (raise fibrinogen } 70-100 \mathrm{mg} / \mathrm{dL} \text { ). }\end{array}$ \\
\hline No demonstrable clot & $\begin{array}{l}\text { Heparin infusion } 8 \text { units } / \mathrm{kg} / \mathrm{h}, \\
\text { no bolus } \\
\text { aPTT goal }=35-45 \mathrm{~s} .\end{array}$ \\
\hline Demonstrable clot & $\begin{array}{l}\text { Heparin infusion } 14 \mathrm{units} / \mathrm{kg} / \mathrm{h}, \\
\text { no bolus } \\
\text { aPTT goal }=45-60 \mathrm{~s} .\end{array}$ \\
\hline
\end{tabular}

Abbreviations: aPTT, activated partial thromboplastin time; CCT, common coagulation test; COVID-19, coronavirus disease 2019; ICU, intensive care unit; MA, maximum amplitude; R, reaction time; TEG, thromboelastography.

Note: For $R>25 \mathrm{~min}, \mathrm{MA}<40 \mathrm{~mm}$, fibrinogen $<150 \mathrm{mg} / \mathrm{dL}$, or aPTT $>70$ s, contact COVID-19 coagulopathy consultant for dose adjustment.

our institution. The CCTs and TEG were available 24 hours a day, 7 days a week, and therefore were primarily used in this group of patients who required intense laboratory evaluation of hemostatic integrity during anticoagulation. The aPTT is
Table 3 Medians, interquartile ranges, and Mann-Whitney test $p$-values for all the studied parameters across the two groups

\begin{tabular}{|l|l|l|l|}
\hline Parameter & $\begin{array}{l}\text { Bleeding } \\
\text { group, } \\
\text { median (IQR) }\end{array}$ & $\begin{array}{l}\text { Nonbleeding } \\
\text { group, } \\
\text { median (IQR) }\end{array}$ & $p$-Value \\
\hline$R(\mathrm{~min})$ & $26.00(22.95)$ & $6.80(4.50)$ & 0.0001 \\
\hline MA (mm) & $55.35(22.88)$ & $54.20(12.00)$ & 0.36 \\
\hline$k(\mathrm{~min})$ & $8.85(16.43)$ & $1.80(0.60)$ & 0.0002 \\
\hline$\alpha($ degrees) & $23.25(43.43)$ & $63.10(5.30)$ & 0.0001 \\
\hline PT (s) & $12.0(2.0)$ & $10.9(0.8)$ & 0.0013 \\
\hline aPTT (s) & $73.1(41.3)$ & $36.8(11.0)$ & 0.0006 \\
\hline $\begin{array}{l}\text { Fibrinogen } \\
(\mathrm{mg} / \mathrm{dL})\end{array}$ & $220.0(61.3)$ & $314.8(99.8)$ & 0.0019 \\
\hline $\begin{array}{l}\text { D-dimer } \\
(\mu \mathrm{g} / \mathrm{dL})\end{array}$ & $1.30(1.17)$ & $1.50(2.00)$ & 0.70 \\
\hline $\begin{array}{l}\text { Platelet count } \\
\left(\times 10^{3} / \mathrm{mm}^{3}\right)\end{array}$ & $169.0(93.5)$ & $156.0(148.0)$ & 0.69 \\
\hline
\end{tabular}

Abbreviations: aPTT, activated partial thromboplastin time; IQR, interquartile range; $k$, clot formation time; MA, maximum amplitude; $n$, sample size; PT, prothrombin time; $R$, reaction time.

Note: The $R, k, \alpha$ angle, PT, aPTT, and fibrinogen were statistically significant predictors of bleeding.

not as sensitive an indicator of LMWH efficacy as of UFH. ${ }^{16}$ Moreover, the inclusion of enoxaparin in the control group confounds the data. However, the presence of enoxaparin in the nonbleeding group has resulted in a lower aPTT than if only UFH patients had been included. 
This analysis reflects our initial experience with COVID19 ICU patients when the algorithms were under development. We now use UFH for ICU anticoagulation because of the ease in tracking anticoagulation with TEG, aPTT, and fibrinogen, as well as the potential antiviral and anti-inflammatory effect of the UFH molecule for COVID-19 patients. A future study comprising a larger cohort of patients only treated with UFH is underway.

\section{Conflict of Interest}

E.M., H.M., and M.M.W. report research grants from Haemonetics Inc. Boston, MA, outside the submitted work.

\section{Acknowledgments}

The authors would like to thank Shareen Lee, ASC MBA and Susan Richeson, RN MBA for their laboratory direction in the use of thromboelastography.

\section{References}

1 Al-Samkari H, Karp Leaf RS, Dzik WH, et al. COVID-19 and coagulation: bleeding and thrombotic manifestations of SARSCoV-2 infection. Blood 2020;136(04):489-500

2 Thachil J, Agarwal S. Understanding the COVID-19 coagulopathy spectrum. Anaesthesia 2020;75(11):1432-1436

3 Panigada M, Bottino N, Tagliabue $\mathrm{P}$, et al. Hypercoagulability of COVID-19 patients in intensive care unit: a report of thromboelastography findings and other parameters of hemostasis. J Thromb Haemost 2020;18(07):1738-1742

4 Thachil J, Tang N, Gando S, et al. ISTH interim guidance on recognition and management of coagulopathy in COVID-19. J Thromb Haemost 2020;18(05):1023-1026

5 Cohoon KP, Mahé G, Tafur AJ, Spyropoulos AC. Emergence of institutional antithrombotic protocols for coronavirus 2019. Res Pract Thromb Haemost 2020;4(04):510-517

6 Barrett CD, Moore HB, Yaffe MB, Moore EE. ISTH interim guidance on recognition and management of coagulopathy in COVID-19: a comment. J Thromb Haemost 2020;18(08):2060-2063

7 Tang N, Bai H, Chen X, Gong J, Li D, Sun Z. Anticoagulant treatment is associated with decreased mortality in severe coronavirus disease 2019 patients with coagulopathy. J Thromb Haemost 2020;18(05):1094-1099

8 Paranjpe I, Fuster V, Lala A, et al. Association of treatment dose anticoagulation with in-hospital survival among hospitalized patients with COVID-19. J Am Coll Cardiol 2020;76(01):122-124

9 Chick D. American College of Physicians. Treatment: pharmacotherapy overview. In: COVID-19: an ACP Physician's Guide \& Resources. Philadelphia, PA: American College of Physicians; 2020
10 Chaudhary R, Kreutz RP, Bliden KP, Tantry US, Gurbel PA. Personalizing antithrombotic therapy in COVID-19: role of thromboelastography and thromboelastometry. Thromb Haemost 2020; 120(11):1594-1596

11 Collett LW, Gluck S, Strickland RM, Reddi BJ. Evaluation of coagulation status using viscoelastic testing in intensive care patients with coronavirus disease 2019 (COVID-19): an observational point prevalence cohort study. Aust Crit Care 2020 (e-pub ahead of print). Doi: 10.1016/j.aucc.2020.07.003

12 Yuriditsky E, Horowitz JM, Merchan C, et al. Thromboelastography profiles of critically Ill patients with coronavirus disease 2019. Crit Care Med 2020;48(09):1319-1326

13 Fogarty PF, Tarantino MD, Brainsky A, Signorovitch J, Grotzinger KM. Selective validation of the WHO Bleeding Scale in patients with chronic immune thrombocytopenia. Curr Med Res Opin 2012;28(01):79-87

14 White H, Sosnowski K, Bird R, Jones M, Solano C. The utility of thromboelastography in monitoring low molecular weight heparin therapy in the coronary care unit. Blood Coagul Fibrinolysis 2012;23(04):304-310

15 Smythe MA, Priziola J, Dobesh PP, Wirth D, Cuker A, Wittkowsky AK. Guidance for the practical management of the heparin anticoagulants in the treatment of venous thromboembolism. J Thromb Thrombolysis 2016;41(01):165-186

16 Hoffman M. Heparins: clinical use and laboratory monitoring. Lab Med 2010;41(10):621-626

17 Barnes GD, Burnett A, Allen A, et al. Thromboembolism and anticoagulant therapy during the COVID-19 pandemic: interim clinical guidance from the anticoagulation forum. J Thromb Thrombolysis 2020;50(01):72-81

18 Moores LK, Tritschler T, Brosnahan S, et al. Prevention, diagnosis, and treatment of VTE in patients with coronavirus disease 2019: CHEST Guideline and Expert Panel Report. Chest 2020;158(03): 1143-1163

19 Desborough MJR, Doyle AJ, Griffiths A, Retter A, Breen KA, Hunt BJ. Image-proven thromboembolism in patients with severe COVID19 in a tertiary critical care unit in the United Kingdom. Thromb Res 2020;193:1-4

20 Fraissé M, Logre E, Pajot O, Mentec H, Plantefève G, Contou D. Thrombotic and hemorrhagic events in critically ill COVID-19 patients: a French monocenter retrospective study. Crit Care 2020;24(01):275

21 Musoke N, Lo KB, Albano J, et al. Anticoagulation and bleeding risk in patients with COVID-19. Thromb Res 2020;196:227-230

22 Colman E, Yin EB, Laine G, et al. Evaluation of a heparin monitoring protocol for extracorporeal membrane oxygenation and review of the literature. J Thorac Dis 2019;11(08):3325-3335

23 Chlebowski MM, Baltagi S, Carlson M, Levy JH, Spinella PC. Clinical controversies in anticoagulation monitoring and antithrombin supplementation for ECMO. Crit Care 2020;24(01):19

24 Mulder MMG, Fawzy I, Lance MD. ECMO and anticoagulation: a comprehensive review. Neth J Crit Care 2017;26(01):6-13 